\title{
Effect on dynamic postural stability after cold therapy application to the knee joint: a pilot randomized controlled trial
}

\author{
DOI: https://doi.org/10.5114/pq.2021.105884
}

\author{
Sudip Debnath ${ }^{1}$, Asir John Samuel ${ }^{2}$ \\ 1 Tripura Institute of Paramedical Sciences, Hapania, Amtali, Tripura, India \\ ${ }^{2}$ Department of Pediatric and Neonatal Physiotherapy, Maharishi Markandeshwar Institute of Physiotherapy \\ and Rehabilitation, Maharishi Markandeshwar (Deemed to be University), Mullana, Haryana, India
}

\section{Abstract}

Introduction. In addition to the merits of cold therapy in the management of soft tissue injuries, it decreases dynamic stability. Controversies exist regarding this statement. Thus, the aim of the study was to find the effect on postural stability after the application of cold therapy to knee joint.

Methods. Overall, 24 individuals aged 18-25 years participated in this pilot study. They were randomly divided into 2 groups, experimental and control group. Each participant in the experimental group was asked to perform the Star Excursion Balance Test (SEBT) under 2 conditions, before and after the application of cold therapy. The subjects received ice pack wrapping of the knee for 10 minutes. The control group performed SEBT at room temperature with an interval of 10 minutes. The 2 groups were analysed and compared.

Results. The demographic characteristics of the participants recruited were as follows: age: 21 (20.2-21.9) years, height: 166.3 $(162.9-169.7) \mathrm{cm}$, weight: $61.2(56.7-65.7) \mathrm{kg}$, and BMl: $22.06(20.7-23.4) \mathrm{kg} / \mathrm{cm}^{2}$. There was no significant difference $(p \geq 0.05)$ between SEBT scores between the experimental group and the control group.

Conclusions. Cold therapy did not have any influence on dynamic postural stability among college students if applied over the knee joint for 10 minutes.

Key words: ice, cryotherapy, soft tissue injuries, students, temperature

\section{Introduction}

Cold therapy is a treatment modality that physiotherapists use in patients to facilitate fast recovery from soft tissue injuries [1]. Its application leads to a decrease in tissue temperature. Cold therapy has been mainly used as an immediate treatment option to reduce the pain due to soft tissue injuries. Physiological changes resulting from cold therapy for any kind of traumatic injury have been reported. Cold therapy facilitates reduction of oedema, swelling, temperature, and inflammation [1]. Though it has many known benefits, some investigators have reported decreased functional performance after its therapeutic application [1, 2]. Cold therapy decreased the tissue temperature and contusion, but much controversy remains regarding the clinical practice [3]. Studies have shown that cold therapy application alters proprioception [3]. The application of cold therapy has negative effects on lower limb muscle power as evaluated by vertical-jump height and agility performance [4].

Cold therapy exerts negative effects mainly in the knee joint positional sense [1]. Its application to the knee joint for 15 minutes increased knee joint stiffness and decreased joint position sense acuity [3]. Fullam et al. [1] reported that the control of sensorimotor phenomena in the knee joint, which was quantified by postural stability (static), was negatively affected by more than 15 minutes of cooling procedure known as hypothermia anaesthesia, a method similar to cold therapy application. Even the ability to maintain standing balance is decreased after the application of cold therapy by reducing dynamic stability [4]. The effect of balance (dynamic standing) better represents the appropriate demands of the lower extremity during functional tasks [1]. Postural stability was evaluated in the dynamic standing position, which was quantified by the Star Excursion Balance Test (SEBT) [5]. It was hypothesized that cold therapy had an effect on postural control [4]. SEBT refers to the demands of the neuromuscular system and measures dynamic postural control [6]. The neuromuscular factor of dynamic postural stability can be used as a clinical measure to predict the risk of future injury.

Even though the application cold therapy exerts deleterious effects on balance and proprioception, it still remains the standard treatment immediately after soft tissue injuries. The risk-benefit ratio is outweighed and this has led to a reinvestigation of the hypothesis on cold therapy. Here, we hypothesized that there existed no significant difference in the dynamic stability after the application cold therapy over the knee joint as compared with the control. Thus, the objective of the study was to examine the dynamic postural stability after cold therapy application.

\section{Subjects and methods}

\section{Participant recruitment}

A total of 24 volunteering collegiate male and female students aged 18-25 years were recruited from the recognized

Correspondence address: Asir John Samuel, Department of Pediatric and Neonatal Physiotherapy, Maharishi Markandeshwar Institute of Physiotherapy and Rehabilitation, Maharishi Markandeshwar (Deemed to be University), Mullana-133207, Haryana, India, e-mail: asirjohnsamuel@mmumullana.org

Received: 31.03 .2020

Accepted: 12.05 .2020

Citation: Debnath S, Samuel AJ. Effect on dynamic postural stability after cold therapy application to the knee joint: a pilot randomized controlled trial. Physiother Quart. 2021;29(3):19-23; doi: https://doi.org/10.5114/pq.2021.105884. 
university population for a pretest-posttest control group design study after screening 28 students by using a simple random sampling method with online random number generators (https://www.random.org/integer-sets/). Individuals who had experienced any lower limb fracture within the previous 6 months or had a skin disease or any other known disease which might prevent them from taking part in the study were excluded.

\section{Participant allocation}

All the required anthropometrics such as height, weight, and limb length were measured before the beginning of the trial. After the recruitment, 24 participants were randomly divided into 2 groups, namely the experimental and the control group, by using an online block randomization portal (https://www.sealedenvelope.com/simple-randomiser/v1/ lists). We used 6 blocks with 4 rows, producing a $6 \times 4$ matrix. Sequentially numbered opaque sealed envelopes were used to conceal allocation. Each participant was asked to pick an envelope from a stack just before the trial. In this way, allocation bias was controlled.

\section{Experimental group}

Before the test session, SEBT was demonstrated to all the participants by the principal investigator, and 4 practice trials were allowed in each direction, as advocated by Robinson and Gribble [7]. Skin temperature was measured with a calibrated digital thermometer before and after cold therapy application by the principal investigator. Both the dominant and non-dominant lower extremities were tested; their sequence was randomized by tossing a coin (head: dominant leg; tail: non-dominant leg). SEBT was performed in the following order: anterior, anteromedial, medial, posteromedial, posterior, posterolateral, lateral, and anterolateral directions. The participants initially stood with their big toes positioned at the centre of a grid laid on the laboratory floor and extend- ing from the 8 reach directions. After the completion of one leg evaluation, another leg was measured with the same procedure. A $1.5-\mathrm{m}$ measuring tape allowed us to easily quantify the reach distance scores in each direction. Reach distances were measured from the centre of the grid to the point of maximum reach, which was observed visually and noted by the principal investigator. Immediately after the completion of SEBT, the cooling procedure for the knee joint was initiated by using a cryopack or cold pack of 4 " $\times 10$ " in dimension (3M Nexcare ${ }^{\text {TM }}$ Reusable Cold/Hot Pack). The reusable cold pack has a blue gel in a sealed bag. The components of the blue gel are primarily water (60-70\%), propylene glycol (20$35 \%)$, and sodium carboxymethyl cellulose $(<5 \%)$. The participants were asked to lie supine and the investigator applied the cold therapy for 10 minutes. Two cold packs were used to cover a single knee joint in an anterior-posterior fashion. After the 10-minute period of cold therapy, the cryopack was removed, and the participants performed SEBT for the second time. Three consecutive trials in each direction were implemented at 2 time points: before and after the cold therapy. The same investigator was responsible for the precold therapy and post-cold therapy SEBT and skin temperature measurements. Throughout the procedure, room temperature was maintained at $27^{\circ} \mathrm{C}$.

\section{Control group}

The participants in the control group performed SEBT as mentioned above; then, after 10 minutes of rest, the second SEBT was completed. The 10-minute rest corresponded with the duration of cold therapy application. During the period, the subjects were asked to sit comfortably in a wooden chair with their back and arms in a resting position. The modified CONSORT flow diagram for individual randomized controlled trials of nonpharmacologic treatments (CONSORT-NPT) for the study is displayed in Figure 1. This intervention satisfied the template for intervention description and replication (TIDieR) checklist.

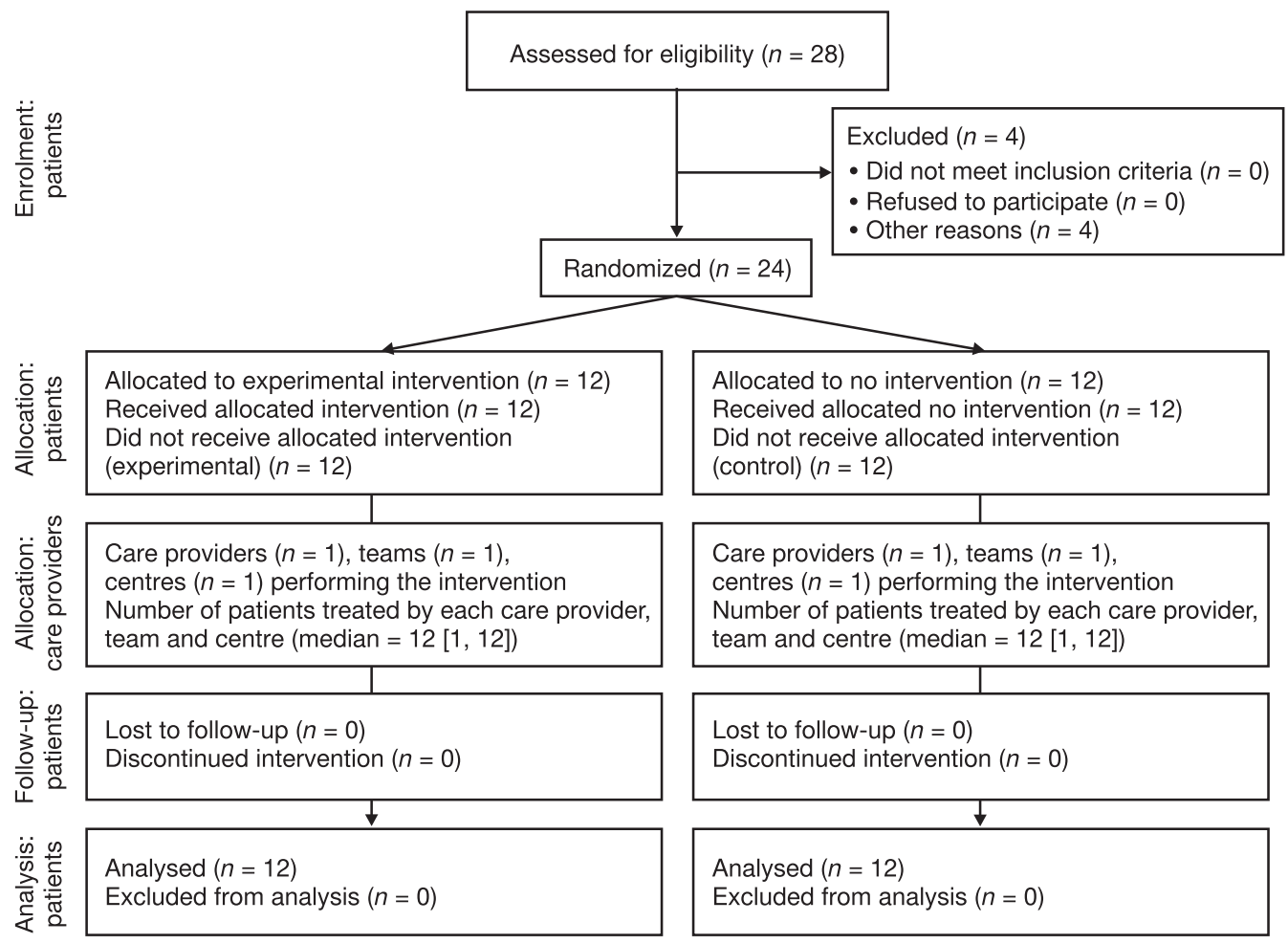




\section{Data analysis}

The statistical analysis of the collected data was performed with the Statistical Package for the Social Sciences software $\left(\right.$ IBM $^{\circledR}$ SPSS $^{\circledR}$, version 20.0, IBM Corp., Armonk, USA) for Windows 10 Home Edition. The normal Gaussian distribution of the collected data was verified by the ShapiroWilk test. As the data followed the normal distribution, the descriptive statistics were expressed in terms of mean \pm standard deviation $(S D)$. Demographic differences between the experimental group and the control group were explored by a statistical test of significance, independent $t$-test. Mean SEBT values for 8 directions were reported as mean $\pm S D$, and differences between the 2 groups in the pre- and postintervention status were established by an independent $t$-test. A paired $t$-test explored within-group differences. The mean changes of score between the groups were compared by using an independent $t$-test. The alpha value for all the analyses was set at the significance of less than 0.05 to minimize type I error. We used the sample size of 12 in each group, as it was sufficient for a pilot study [8]. The effect size (ES) was calculated by Cohen's $d$ by using the following formula [9]:

$$
(M 1-M 2) \div \sqrt{ }\left[(S D 2)^{2}+(S D 1)^{2}\right] / 2
$$

As recommended, if $E S$ was $\geq 0.2, \geq 0.5$, and $\geq 0.8$, then it was considered as small, medium, and large, respectively $[10,11]$. The retrospective (post-hoc) power analysis was performed by using the mean difference between 2 dependent

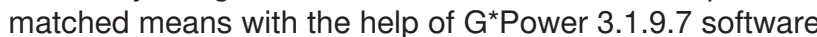
for Windows X (Heinrich-Heine-Universität Düsseldorf, Düsseldorf, Germany; http://www.gpower.hhu.de/) [12, 13]. As indicated in a previous research on SEBT, the minimal detectable change (MDC) in SEBT is between 6.68 and $9.15 \mathrm{~cm}$ [14]. Therefore, we calculated the clinically significant difference between the groups with the lower limit of MDC $(6.68 \mathrm{~cm})$.

\section{Ethical approval}

The research related to human use has complied with all the relevant national regulations and institutional policies, has followed the tenets of the Declaration of Helsinki and the Indian Council of Medical Research's National Ethical Guidelines for biomedical and health research involving human participants as of 2017, and has been approved by the student project committee of Maharishi Markandeshwar Institute of Physiotherapy and Rehabilitation (MMIPR/SPCA/ 17/14). The study protocol was registered prospectively under the International Clinical Trials Registry Platform of the World Health Organization with the recognized primary clinical trial registry ClinicalTrials.gov identifier of NCT03151031 on May 12, 2017.

\section{Informed consent}

Informed consent has been obtained from all individuals included in this study.

\section{Results}

From among the screened 28 college students, 24 were recruited and completed the trial, with no drop-outs due to the 10-minute intervention. None of them complained of discomfort during the test. The demographic characteristics of the participants are presented in Table 1. Here, no significant differences were observed between the experimental group and the control group. Thus, we can say that the groups were matched in their age, weight, and height, which improves the quality of the study. The pre- and post-intervention skin temperatures are depicted in Table 2. Tables 3 and 4 show SEBT scores in the 2 groups, with mean, $S D$, $p$-value, ES by Cohen's $d$ formula, and retrospective (post-hoc) power analysis of the pre-post-intervention changes. The mean changes between the pre- and post-intervention SEBT scores in both groups are displayed in Table 5 . In both groups, the mean SEBT score change was less than $6.68 \mathrm{~cm}$. Thus, no meaningful detectable changes occurred in the groups.

\section{Discussion}

In the present study, 24 individuals were included and baseline scores of SEBT reaching distance in each direction were established. The subjects were randomly divided into 2 groups, 12 individuals each. In the experimental group, cold therapy application was performed for 10 minutes and then knee joint temperature was taken and SEBT measurement was obtained. In the control group, knee joint temperature was taken and then postural stability in 8 different directions was evaluated with SEBT. The findings of the current study confirmed no significant MDC differences in SEBT reach distances between the 2 groups after the 10-minute intervention. Hence, the study confirmed the null hypothesis.

One similar study was conducted by Fullam et al. [1], in which deficits in dynamic postural stability were reported after cold therapy application to the ankle joint. After the intervention, the reach in 3 specific directions was measured by using

Table 1. Demographic characteristics of the recruited college students

\begin{tabular}{|l|c|c|}
\hline Characteristic & Experimental group & \multicolumn{1}{|c|}{$p$} \\
\hline Age (years) & $21.8(20.4-20.3)$ & $20.2(19.3-21.1)$ \\
\hline Height $(\mathrm{cm})$ & $166.5(161.03-171.9)$ & $166.1(161.4-170.9)$ \\
\hline Weight $(\mathrm{kg})$ & $61.5(56.2-66.7)$ & $60.9(52.6-69.2)$ \\
\hline Body mass index $\left(\mathrm{kg} / \mathrm{cm}^{2}\right)$ & $22.2(20.4-23.9)$ & $21.9(19.4-24.3)$ \\
\hline
\end{tabular}

Table 2. Skin temperature $\left({ }^{\circ} \mathrm{C}\right)$ in the recruited college students

\begin{tabular}{|l|c|c|}
\hline Temperature & Experimental group & Control group \\
\hline Before cold therapy & $41.6(40.8-42.2)$ & 0.24 \\
\hline After cold therapy & $34(33.8-34.4)$ & $41.1(39.2-41.9)$ \\
\hline$p$ & 0.002 & $40.6(37.9-41.2)$ \\
\hline
\end{tabular}


Table 3. Star Excursion Balance Test scores in the experimental group

\begin{tabular}{|l|c|c|c|c|c|}
\hline Reaching direction & Before CT & After CT & Effect size & Power & $p$ \\
\hline Anterior reach $(\mathrm{cm})$ & $75.5 \pm 10.4$ & $77.1 \pm 9.84$ & 0.1 & $6 \%$ & 0.16 \\
\hline Anteromedial reach $(\mathrm{cm})$ & $75.7 \pm 11.1$ & $76.1 \pm 10.6$ & 0.1 & $6 \%$ & 0.6 \\
\hline Medial reach $(\mathrm{cm})$ & $74.8 \pm 11.3$ & $77.4 \pm 12.3$ & 0.2 & $9 \%$ & 0.13 \\
\hline Posteromedial reach $(\mathrm{cm})$ & $74.1 \pm 9.0$ & $73.2 \pm 9.5$ & -0.1 & $-6 \%$ & 0.50 \\
\hline Posterior reach $(\mathrm{cm})$ & $66 \pm 10.1$ & $70.5 \pm 10.9$ & 0.4 & $24 \%$ & 0.02 \\
\hline Posterolateral reach $(\mathrm{cm})$ & $61.2 \pm 8.8$ & $66.3 \pm 9.5$ & 0.6 & $47 \%$ & 0.01 \\
\hline Lateral reach $(\mathrm{cm})$ & $59.5 \pm 9.5$ & $62.1 \pm 9.3$ & 0.3 & $16 \%$ & 0.04 \\
\hline Anterolateral reach $(\mathrm{cm})$ & $72.6 \pm 10.6$ & $74.9 \pm 11.1$ & 0.2 & $9 \%$ & 0.08 \\
\hline
\end{tabular}

CT - cold therapy

Table 4. Star Excursion Balance Test scores in the control group

\begin{tabular}{|c|c|c|c|c|c|}
\hline Reaching direction & Before rest & After rest & Effect size & Power & $p$ \\
\hline Anterior reach $(\mathrm{cm})$ & $67.3 \pm 12.4$ & $70.1 \pm 10.9$ & 0.2 & $9 \%$ & 0.12 \\
\hline Anteromedial reach $(\mathrm{cm})$ & $70 \pm 13.2$ & $71.4 \pm 11.3$ & 0.1 & $6 \%$ & 0.60 \\
\hline Medial reach $(\mathrm{cm})$ & $68.1 \pm 12.9$ & $71.4 \pm 11.1$ & 0.2 & $9 \%$ & 0.05 \\
\hline Posteromedial reach (cm) & $64.5 \pm 13.1$ & $66.8 \pm 12.1$ & 0.2 & $9 \%$ & 0.04 \\
\hline Posterior reach $(\mathrm{cm})$ & $57.1 \pm 14.4$ & $61.3 \pm 12.5$ & 0.3 & $16 \%$ & 0.04 \\
\hline Posterolateral reach $(\mathrm{cm})$ & $52.2 \pm 11.1$ & $56.8 \pm 11.2$ & 0.4 & $24 \%$ & 0.01 \\
\hline Lateral reach $(\mathrm{cm})$ & $45.6 \pm 10.1$ & $50.7 \pm 9.7$ & 0.5 & $35 \%$ & 0.20 \\
\hline Anterolateral reach (cm) & $63.8 \pm 10.3$ & $67.5 \pm 11.3$ & 0.3 & $16 \%$ & 0.50 \\
\hline
\end{tabular}

Table 5. Star Excursion Balance Test mean score changes

\begin{tabular}{|l|c|c|c|}
\hline Reaching direction & Experimental group & Control group & $p$ \\
\hline Anterior reach $(\mathrm{cm})$ & $1.5 \pm 5.28$ & $2.7 \pm 5.3$ & 0.58 \\
\hline Anteromedial reach $(\mathrm{cm})$ & $0.3 \pm 5.4$ & $1.4 \pm 5.9$ & 0.66 \\
\hline Medial reach $(\mathrm{cm})$ & $2.5 \pm 5.3$ & $2.8 \pm 4.5$ & 0.90 \\
\hline Posteromedial reach $(\mathrm{cm})$ & $-0.9 \pm 4.8$ & $2.3 \pm 3.9$ & 0.08 \\
\hline Posterior reach $(\mathrm{cm})$ & $4.5 \pm 6.04$ & $4.2 \pm 6.1$ & 0.90 \\
\hline Posterolateral reach $(\mathrm{cm})$ & $5.1 \pm 5.04$ & $4.5 \pm 4.7$ & 0.79 \\
\hline Lateral reach $(\mathrm{cm})$ & $2.6 \pm 4.1$ & $5.08 \pm 6.8$ & 0.29 \\
\hline Anterolateral reach $(\mathrm{cm})$ & $2.2 \pm 3.7$ & $3.6 \pm 6.1$ & 0.50 \\
\hline
\end{tabular}

SEBT. The authors observed that dynamic postural stability was adversely affected in the participants. Their findings were not consistent with the results of the current study, which showed no statistically significant differences in reach distances after cold therapy application.

A review was carried out by Furmanek et al. [3] to find the effects of cold therapy on the proprioceptive system. They made an extensive literature review to determine the effects of cold therapy on various aspects of proprioception and concluded that there were no reports showing a relation between cold therapy and proprioception [3]. This finding is consistent with the results of the current study, in which cooling the knee joint showed no impact on the postural stability and hence the balance remained unaffected.

Douglas et al. [2] reported a decreased medial and lat- cold therapy application to the ankle joint. They cautioned against an immediate return to sports activities after 15 minutes of cold therapy application. This study again contradicts the findings of the present study. However, we have demonstrated that 10 minutes of cold therapy application to the knee joint does not have any impact on dynamic balance.

To sum up, cold therapy of 10 minutes is safe to be applied over the knee joint during any sports activity in the case of any soft tissue injuries. As it does not influence dynamic balance, athletes can return to their sports activity immediately after the cold therapy session.

\section{Limitations}

Our study had some limitations. These included the unavoidable human error during the documentation of the reach 
distance, insufficient power of the study to confirm the findings, a small sample size (pilot study) [8], and no documentation of temperature change at the tissue level. However, we have documented the skin temperature. Further, the study could be replicated with longer durations of cold therapy, with bigger samples, and with various sports populations as a randomized controlled trial.

\section{Conclusions}

Cold therapy applied over the knee joint for 10 minutes did not have any influence on dynamic postural stability among college students.

\section{Acknowledgements}

The authors address special thanks to Dr. Vencita Priyanka Aranha, MPT (PhD), Research Scholar, Maharishi Markandeshwar Institute of Physiotherapy and Rehabilitation, Maharishi Markandeshwar (Deemed to be University), for the manuscript technical editing.

\section{Disclosure statement}

No author has any financial interest or received any financial benefit from this research.

\section{Conflict of interest}

The authors state no conflict of interest.

\section{Funding}

The study received no funding.

\section{References}

1. Fullam K, Caulfield B, Coughlan GF, McGroarty M, Delahunt E. Dynamic postural-stability deficits after cryotherapy to the ankle joint. J Athl Train. 2015;50(9):893904; doi: 10.4085/1062-6050-50.7.07.

2. Douglas M, Bivens S, Pesterfield J, Clemson N, Castle W, Sole $\mathrm{G}$, et al. Immediate effects of cryotherapy on static and dynamic balance. Int J Sports Phys Ther. 2013;8(1): 9-14.

3. Furmanek MP, Słomka K, Juras G. The effects of cryotherapy on proprioception system. Biomed Res Int. 2014; 2014:696397; doi: 10.1155/2014/696397.

4. Ageberg E, Roberts D, Holmström E, Fridén T. Balance in single-limb stance in healthy subjects - reliability of testing procedure and the effect of short-duration submaximal cycling. BMC Musculoskelet Disord. 2003;4: 14; doi: 10.1186/1471-2474-4-14.

5. Delahunt E, Chawke M, Kelleher J, Murphy K, Prendiville A, Sweeny L, et al. Lower limb kinematics and dynamic postural stability in anterior cruciate ligament-reconstructed female athletes. J Athl Train. 2013;48(2): 172-185; doi: 10.4085/1062-6050-48.2.05.

6. Hart JM, Kuenze CM, Diduch DR, Ingersoll CD. Quadriceps muscle function after rehabilitation with cryotherapy in patients with anterior cruciate ligament reconstruction. J Athl Train. 2014;49(6):733-739; doi: 10.4085/10626050-49.3.39.

7. Robinson RH, Gribble PA. Support for a reduction in the number of trials needed for the star excursion balance test. Arch Phys Med Rehabil. 2008;89(2):364-370; doi: 10.1016/j.apmr.2007.08.139.

8. Julious SA. Sample size of 12 per group rule of thumb for a pilot study. Pharm Stat. 2005;4(4):287-291; doi: 10.1002/ pst.185.
9. Marfo P, Okyere GA. The accuracy of effect-size estimates under normals and contaminated normals in meta-analysis. Heliyon. 2019;5(6):e01838; doi: 10.1016/j. heliyon.2019.e01838.

10. Cohen J. A power primer. Psychol Bull. 1992;112(1): 155-159; doi: 10.1037/0033-2909.112.1.155.

11. Portney LG, Gross KD. Measurement revisited: reliability and validity statistics. In: Portney LG (ed.), Foundaitons of clinical research: applications to evidence-based practice, $4^{\text {th }}$ ed. Philadelphia: FA Davis Company; 2020; 486-508.

12. Peacock JL, Kerry SM, Balise RR. Writing a research protocol. In: Peacock JL, Kerry SM, Balise RR (eds.), Presenting medical statistics from proposal to publication, $2^{\text {nd }}$ ed. Oxford: Oxford University Press; 2017; 22-38.

13. Faul F, Erdfelder E, Lang A-G, Buchner A. G*Power 3: a flexible statistical power analysis program for the social, behavioral, and biomedical sciences. Behav Res Methods. 2007;39(2):175-191; doi: 10.3758/BF03193146.

14. Hyong $\mathrm{IH}$, Kim JH. Test of intrarater and interrater reliability for the Star Excursion Balance Test. J Phys Ther Sci. 2014;26(8):1139-1141; doi: 10.1589/jpts.26.1139. 